\title{
Clothing Colour \& Pattern Recognition for Visually Defective Persons using Grey Level Discrete Wavelet Transform Technique
}

\author{
Vishnuraj. P, D. Vimal Kumar
}

\begin{abstract}
: collecting materials with different colours, patterns are complicated process for visually defective persons. Systematic materials pattern identification is also a denounce investigation highest different pattern deviations. In the study of living human-computer-interaction (HCI) implementation and apply of methods digitalized systems mainly focusing on the particular persons. This system mainly introduces the automatic study on "Blind \& visually defective persons HCI and access to GUI produce a recent scenario imminent solution for visually defective persons and deliver together a new investigation. This Proposed system developed camera-captured on the real cloth identification garments patterns into under 4 diperception (plaid, stripped, pattern less, and 11 garments colours. The camera, a computer, a microphone, and of garments colours. Surrounded upon a pair of images. The garments colours are defined to authorizations. Present, managed by input speech m-phone. To recognize garments patterns, novel RSD and a wavelet sub bands to capture main features of garments patterns. To calculated effectiveness proposed accuracy, for apply various Garments Pattern dataset. Our approaches have $95-98 \%$ recognition accuracy which gathered output performs.
\end{abstract}

Index Terms: Garments pattern identification, dependable cloth system, texture analyze, global and local features, visually defective persons.

\section{INTRODUCTION}

HMI is way in which living humans collaborate in systems else sensory machines and construction functionalities that let humans with systematic in novel basis. As a place of investigation, HCI is placed at the interchange signal processing computer technology, activities sciences, electronics, 3dmedia pasture [1]. Humans communicate or machines in various communication between the both (machines and humans) difficult to insertion. Web and windows applications, browsers, computers, and computeral function make apply GUI today real world.

Major visually defective persons don't have achieved to new teaching aids needed to study. From the data from WHO in Geneva [7], much more 47 million persons world .proposed system HCI can used help of embedded sensors and also devices used visually defective persons. Proposed system targeted these methods of pasture such a pattern identification form of garments a visually defective and colour of garments.

Revised Manuscript Received on April 27, 2020.

* Correspondence Author

Vishnuraj.P*, Research Scholar, Department of Computer Science Nehru Arts \& Science College, Coimbatore, Tamil Nadu-641105

D. Vimal Kumar, Associate Professor, Department of Computer Science Nehru Arts \& Science College, Coimbatore, Tamil Nadu-641105

(C) The Authors. Published by Blue Eyes Intelligence Engineering and Sciences Publication (BEIESP). This is an open access article under the CC BY-NC-ND license (http://creativecommons.org/licenses/by-ncnd $/ 4.0 /)$
Proposed method garments notice garments patterns four deviation. Proposed system is able to detect 11 colours [5]: golden ,orange, red, cyann, ash, purple, and black. Although various methods to the developed for pattern analysis based identical and colour identification in the machine perception based and pictorial processing investigation, identical choices visually defective persons Proposed system develop a machine perception (HCI) prototype to connected combination of pictures of garments for both garments patterns. Configured \& controlling the proposed system, assigning simply speak out words in familiar English to switch on and off the corresponding devices, implementation corresponds functional, adjust. Our various algorithms detect: 1) garments Colours; 2) also the garments are have homogeneous colour 3 ) if colours match for matched database pictures. Proposal HCI camera [2] machanisam to use visually defective persons identification garments patterns and colours. The proposal system contains 3 various components:

1) system like embedded basis surveillance garments earphone for speech wording commands output (Bluetooth or via wifi);

2) information collections (garments) to activity mechanism on machines, garments pattern identification, and colour verification by using a systematic desktop laptop or phone minicomputer or Smartphone.

3) Audio outputs to be identified results in various types of garments patterns and colours.

\section{RELATED WORK}

Dependable garments verification or recognition systems are being developed for different and various garments patterns and color in visually defective persons (blind persons) increase the life standard also safety such persons includes navigation out/indoor using passive RFID and finding route, reading, currency recognition, improvement, and Various more. [10] Advanced functional visually defective persons to select garments defends garments pattern and colours garments shop independently.

Proposed system to unquestionably identification currency note various currency to dependable visually defective persons [2]. This is also a videocamera and computer perception system HCI. Proposed system various features higher rate efficiency, robustness, user friendly use. system prosperous to circumstances occlusion, overturn, crowded environment, change illumination, bills wrinkled, and false identification removal and guide to properly focus bill identification accepting SURF mechanism.

Developed perception equalization machines for [3].

Published By:
Blue Eyes Intelligence Engineering

\& Sciences Publication

(C) Copyright: All rights reserved.

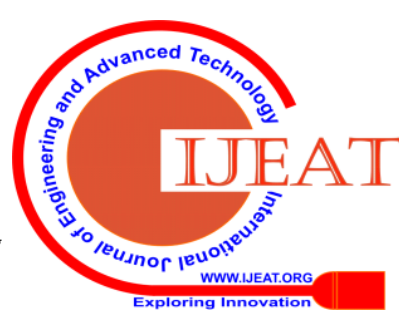


variation of exploration system (different Electronic, (GPS)) from main things in GPS Travel Methods. From the 3 various systems the required of visually defective persons main considered but required to define to the objective dependable garments system in colour visually defective persons.

Proposed a method to attach the Radio Frequency Identification tags to the clothes. Details about garments fetched cloud database maintained by model expertization. Using the various devices, like smart phone laptop etc information about the clothes can be read. Visually defective persons can pick identical clothes with the help of the online stored database. The mearly 189 ten billion persons world today defectives in eye. Admitting to the horde of advanceimprovements functionalities area of astrometry extra surgery, variously these visually defective persons to not never see improvement in their situation. The container Buddy system implemented to allow visuallydefective persons to select out/infits and selected appropriate or closest garments in their garments set. Appropriate GUI interface implemented the visuallydefective, admires selecting to own garments and stuffing to inspire without time. this mechanism depends on tag a radar communication distances, difficult to communicate each devices considered as main drawback of this system [5] proposed system which would permissive to the clothes suitable to values. The system is useful for the person with the normal perception. Identical garments is a denounce process for various visually defective persons. Proposed concept, an ID of concept system to fulfil the type of defects. proposed system methods of 1) a videocamera interfaced to a computer to perform pattern (plaid, stripped, pattern less, and irregular) and colour identical process; 2) voice words proposed system control \& configured; and 3) audio based closed loop to provide identical examines for every colour and patterns of garments. system can shaft garments in deficient colour without pattern garments with various colours and difficult patterns compensation both blind \& colour defective persons. Additionally, our arrangement is prosperous to aberration of contrast, garments rotation and wrinkling. To evaluate the proposed prototype, we collect two denounce databases including Identification [4][12] of garments pattern with reduction set of component. Garments pattern identification denounces methods for blind/visually defective persons. Automatic garments pattern identification also a denounce problem in visually defective persons macinary perception 8system due to the huge pattern deviations and combinations to colour. 4 categories: plaid, stripped, pattern less, and irregular. Because of the [5] [11]. The identification experimental designed data-base with 628 different garments 4 subclassification of pattern. implements methods performs and pictures contextlevel garments identification. Combined SIFT and conversion rtation for texture and pictorial classification. Currently, arrangement depended picture showed various pattern analysis process. proposal represents a huge-set findings and analysis an arrangement that represents pictures as circulation of appearance evoluated from a set sparse of key point located and studies a SVMclassifier with kernels depended on 2 effective measures for matchpoint providers, the Earth Distance movers. [6]. We newer calculated analysis the achievement of our proposed system with various key point identifier and recognition, various plotkernels and differentiation. conduct

a compression with analysis included various stateart identification methods on 4 patterns and 5 object datasets Recognizes garments patterns using SIFT, DWT and Radon Transform feature in four categories and identifies 12 garments colours.[7] City college of New York, America (CCNY) garments pattern dataset[medialab.engr.ccny.cuny.edu/ data is used stripe, plaid, plain and pattern less. For recognition SURF (speedup robust feature), more compact, robust to SIFT [8] [9] is combined with feature extracted by applying GLCM algorithm on images obtained by DWT transform techniques.

\section{SYSTEM OVERVIEW}

The proposed system automatic colourwithpattern identification (strip, plaid, plain and less pattern) different colors ( yellow, green, cyan, and etc.). collecting appropriate colorwithpattern of the garment is complicated the visually defective persons.

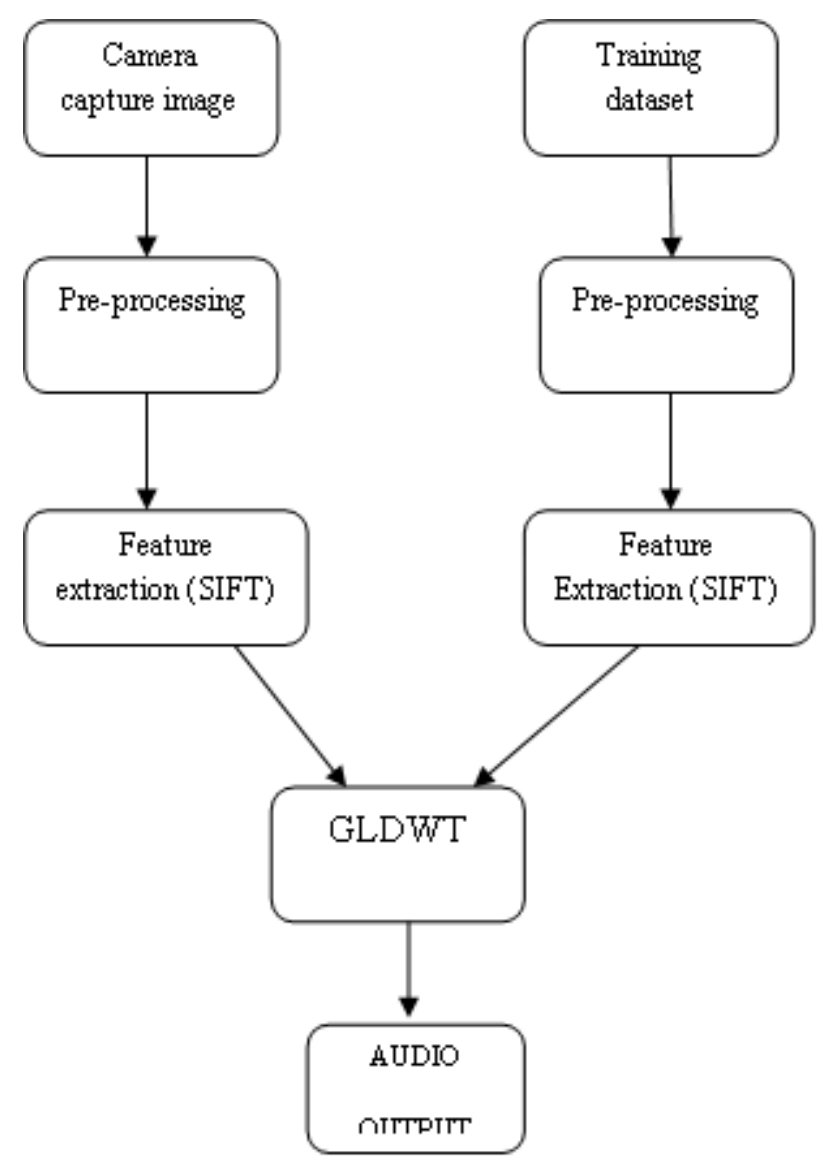

Figure 1: Image Processing Process

The information process consist of on three factors

1) Pre-processing

2) featureextraction

3)classification

The figure 1 describe the proposed system block diagram. Proposed a method to attach the algorithamatic Identification tags to the clothes. Details about garments fetched cloud database maintained by model expertization. Using the various devices, like smart phone laptop etc information about the clothes can be read.

Published By:

Blue Eyes Intelligence Engineering \& Sciences Publication (C) Copyright: All rights reserved.

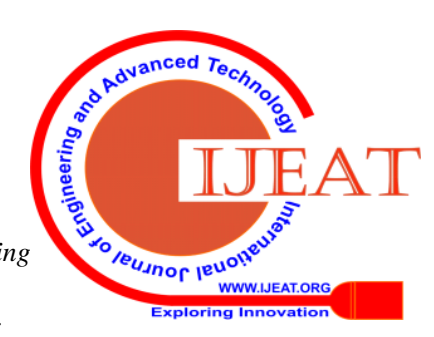


Visually defective persons can pick identical clothes with the help of the online stored database. The identification experimental designed data-base with 628 different garments 4 subclassification of pattern. implements methods performs and pictures contextlevel garments identification. Combined SIFT and conversion rtation for texture and pictorial classification. Currently, arrangement depended picture showed various pattern analysis process. proposal represents a huge-set findings and analysis an arrangement that represents pictures as circulation of appearance evoluated from a set sparse of key point located and studies a SVMclassifier
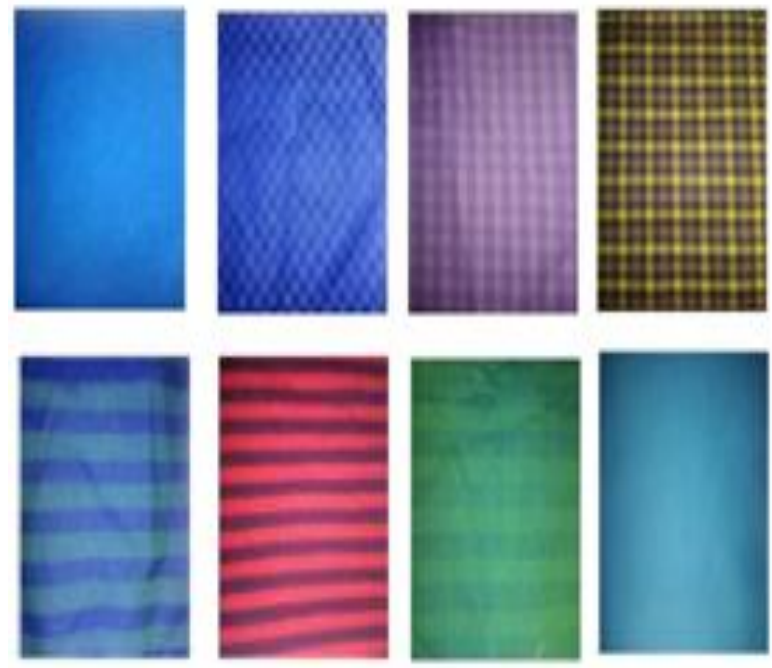

Figure 2: garments pattern samples colour deviations

Conduct a compression with analysis included various stateart identification methods on 4 patterns and 5 object datasets Recognizes garments patterns using SIFT, GLDWT and Radon Transform feature in four categories and identifies 12 garments colours. City college of New York, America (CCNY) garments pattern dataset[medialab.engr.ccny.cuny.edu/ data is used stripe, plaid, plain and pattern less. For recognition SURF (speedup robust feature), more compact, robust to SIFT is combined with feature extracted by applying GLCM algorithm on images obtained by GLDWT transform techniques.

\section{THE PROPOSED FRAMEWORK}

The various Garments patterns present at the visualized to colourwithpatterns few basic primitives (strip, plaid, plain and less pattern). Ever, due to the colour difference, base primitives of the repeted garments (Fig. 2 presents the garments pattern samples colour deviations). Other present evacuation of common values for garments patternwithcolor, i.e,.,

1)Radon Signature used to pre-processing,

2)Gray Level co-occurrence matrix algorithm (GLCM)

3) standard wavelet transform (SWT) algorithm used to feature extraction,

4) SIFT expression apply colour identification, pattern classification done by help of upport vector machine (SVM).

\section{A. Radon Signature Based Pre-Processing}

Garments images present large different deviations As shown in Fig. 2, the garments plaid strip are patterns homogeneous anisotropic. In contrast, basis garments classified pattern less, regular form s hetrogeneiousisotropic patterns. Initially Radon Signature Based Pre-Processing used to gather the information of garments.

\section{B. SIFT Feature Extraction}

Scale Invariance feature method SIFT common image feature extraction. color scale variance difference are used to in this method garments features extracted training garments identified in garment image scale, noiseillumination, of garmnets image invariant scale. Different feature values are extracted in garment images.

\section{GLDWT based training}

The extracted garment images are segments gathered and recognize garmentspatterns with effectively help of SVMs classifier. Once SIFTfeature extracted created output each garmentsvalues images concluded, system continuously observed trained garment values using Multiclass SVM tool box.

classes = svmclassify $($ svmStruct, data(test, $)$,'showplot', true);

Above the Multiclass SVM describe the data to classify the patternwithcolor.

The concluded SIFTfeature merged way minimum rotation same time more garment pictures intensity power. It meaningful the sensitivity of prediction garments prediction depends on the common feature.

\section{classperf(cp,classes,test); $\mathrm{cp}$.CorrectRate}

The SVMs classifiers used in garments patternwithcolor system. SVMs classifiers find a max margin garments image plane space.

\section{EXPERIMENTAL RESULTS AND ANALYSIS}

Classification the results obtained by comparing the train garment image and the test garment image. The trainimage (camera captured garmentimage) attained in the CCNY database. Each picture undergo all the garment image extraction binery values. The input picture given 256X256 pixel. This input picture size modified according the system specification. The proposed system uses dataset of various, dataset includes 25 pictures of 4 different typical garments pattern designs stripe, plaid, plain and pattern less. All pictures are trained values fed to SVMs classifier algorithm. the garmentpatterns differentiated. The proposed systems represented are

(1) Radon Transformation based Pre-processing

(2) Feature extraction-SIFT and SWT

(3) GLDWT classifier cloth classification

\section{A. Garments Colour Identification}

In the proposed system, the hue angle calculated on a colorwheel. complete red hue exact $0^{\circ}$, complete greenhue value are $120^{\circ}$, and complete blueshue value are $240^{\circ}$. V represents brightness. Intensity represents lightness picture color, mentioned integers as the avg red,

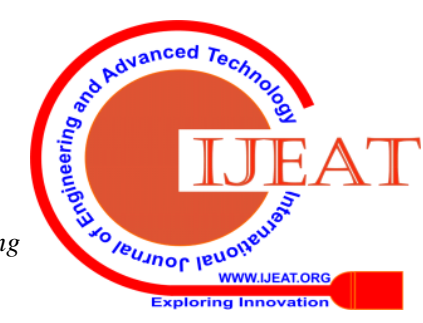


blue and green represents (RGB) measures. HSV, definition of saturation is a measure of a colour's purity/greyness value in image. Purer colours saturation measure closer to 1 , while greyer colours saturation measure closer to 1 . particular, for each of the garments picture, the color identifier SVMs classifies image pixelsmeasures in the picture the following colors: white, black, red, orange, etc.,. Each pictures article garments is first converted from RGB picture to HSV color space picture. Then, HSV cap quantized into minimum measures of colours. If cloth contains differential colors, the most downwards colors outputted. The dominant colours will be communicated in auditory to the visually defective persons.

Proposed feature measures, first calculate and find complementry link activity diagram various feature picture divisional including garment picture features of Radon Signature picture (RadonSig) statistic wavelet (STA) method based analysis,

Table 1 represents various clothes and color as voice output represents in the current model.

TABLE 1: Image Cloth and Colour Identification

\begin{tabular}{|l|l|l|l|l|}
\hline IMAGE & & & & \\
\hline COLOUR & YELLOWBLUE & RED & BLUISH & GREEN BLUE \\
\hline
\end{tabular}

\section{B. Pattern Detection}

\section{Multiple Features}

In order to deal with the various interclass deviations presented in the clothes pattern (plaid, stripped, pattern less, and irregular), global image features and local image structural features are concatenated.

\section{Classification}

Concatenated feature vector is given as input to SVMs (support vector machines). It meaningful reliability and flexibility of performance evaluation output garments picture feature. The context of garments paternwithcolor classification, immediate close to the seperational region interest ROI less reliable than the minimum deep regional boundry in garment pictures. supportvector machines are applied classifier to identification and the garments patterns, color into four various categories (plain, plaid, stripe and pattern less) in our cloth patternwithcolor recognition. SVMs find a maximum hyperplane in the picture margin entire feature space.

Fig 3 represents to the various pattern identification result of pattern identification using SVMs classifier using various cloths, Fig 4 represents to future system model to cloth or product identification system for visually defective persons, inbuilt various sensors like camera voice enunciator and necessary embedded equipped with this system model
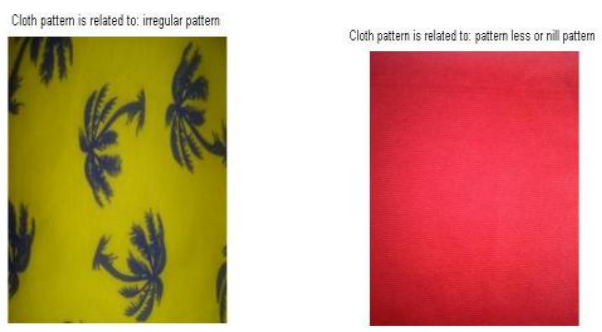
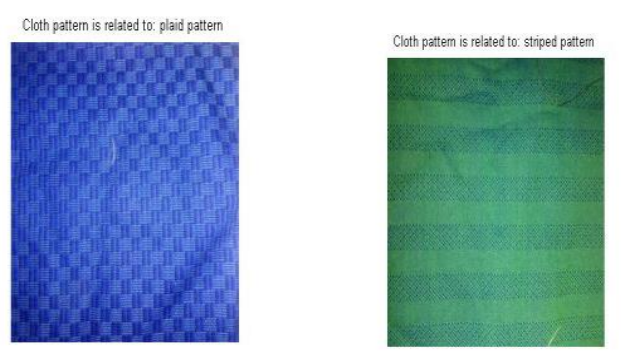

Figure 3: cloth pattern output result (plaid, stripped, pattern less, and irregular)

Table 2: cloth pattern output result compression between existing and proposed

\begin{tabular}{|c|c|c|}
\hline Parameters & $\begin{array}{c}\text { Previous } \\
\text { System, }\end{array}$ & This System \\
\hline Pre-processing & $\begin{array}{c}\text { GREY } \\
\text { conversion and }\end{array}$ & $\begin{array}{c}\text { Radon } \\
\text { transformation }\end{array}$ \\
\hline $\begin{array}{c}\text { Color } \\
\text { identification }\end{array}$ & SIFT,DWT & SIFT,SWT \\
\hline $\begin{array}{c}\text { Pattern } \\
\text { identification }\end{array}$ & $\begin{array}{c}\text { naive Bayes } \\
\text { classifiers, }\end{array}$ & GLDWT \\
\hline Features Used & 9 & 18 \\
\hline \% Accuracy & 96.180 & $95-98$ \\
\hline
\end{tabular}

TEBLE 2 described compression result between the existing and proposed system result and efficiency proposed system more powerful comparing to the existing system where detect cloth pattern and colors.

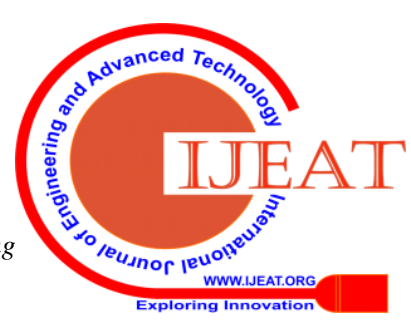




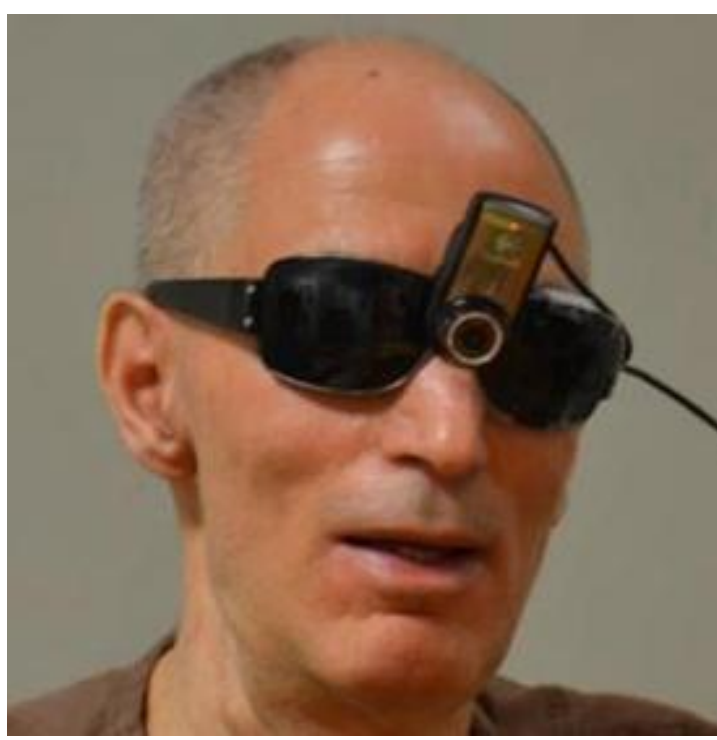

Figure 3: future glass in build system implementation

\section{CONCLUSION}

Proposed paper, novel system identification garments different patterns and colors' to help visually defective persons with our outfit selection in their day today life. The developed prototype has significant detection and identification accuracy and is robust to cloth rotation, cloth illumination, cloth scaling and other such challenges that exist in this domain. Experimental results demonstrate that our proposed novel method is much simpler than Various existing system methods in various garments pattern recognition. Furthermore, the performance evaluation on traditional texture several datasets validates valuable. This investigation contributes to the study of various modifications and to garments. Our investigation proves to be a small contribution towards the process of bringing the blind and the visually-defective persons into the mainstream by providing independence to them and thus uplifting their self-esteem in daily life.

\section{REFERENCES}

1. Xiaodoneg Yeang, Shauai Yeuan, andYingLiTiean, "Dependable Garments Pattern Recognition Visually Defective Persons," IEEE HMI systems, vol. 14, NO.12, 4, 2014.

2. [2] S. Liue, J. Feneg, Z. Songe, H. Lue, C. Xue, and S. Yuaan, "magic closet, tell me what wear," in Proc. Multimedia, 2011.

3. Y.Tiean, X. Yange, C. Yie, and A. Arditih, "Toward computer perception based finding aid for blindpersons to access unfamiliar indoor environments," Appl., vol. 21, no. 2, pp. 511-525, 2011.

4. J.Roshe, "dressing the visually defective persons," In Proceedings of the Southeast conference, pages 601-605., 2008.

5. S.Yuaan, Y.Tiane, and A. Arditih, "Clothes for visually defectivepersons,” J. Technol. 1087-1099.

6. S.Yuaan, Y.Tiane, and "Clothes identical for visually defective persons,” J. Technol. 1017-1037.

7. X.Yange, S.Yuaen, and Y.Tiane, "clothes patterns for blind humans by confidence margin based feature combination," in Proc. Int.ACM Conf. Multimedia, 2012, pp. 1197-1200.

8. J.Zhannge, M.Marszaeleke, S.Lazeebnik, and C.Schimide, "Localfeatures and svmkernels for classification of object categories: comprehensive study," Int. J. Comput. Vis., vol. 63, no. 5, pp. 223-248, 2008.

9. XiaodonYange , ShuaiYuane, and YingliTiane ,"Dependable garments pattern recognition visually defective persons", IEEE HMS ,vol 41,no 4,5, 2015.

10. Herbeert Bay, TinneTutelary, and LucVan Goool. "Surf: Speeded up robust features," in-ECCV 2008, pages 414-427. Springer, 2008.
11. S.Lazebnike, C.Schmide, "ASaparse Texture Representation Using LocalAffineRegions," IEEE Pattern Analysis and Machine Intelligence, vol. 37, no. 18, pp. 1185-1197, 2007.

12. S.Liue, J.Afeng, T.Zhangh, H.Lue, C.Xue, and S.Yuane, "Hi, Magic Closet, Tell Me What to Wear," In Proc. ACM Multimedia, 2014.

13. D.Lowe, "Distinctive Image Features from Scale-invariant Keypoints," International Journal Version, vol. 60, no. 8, pp. 101120, 2006.

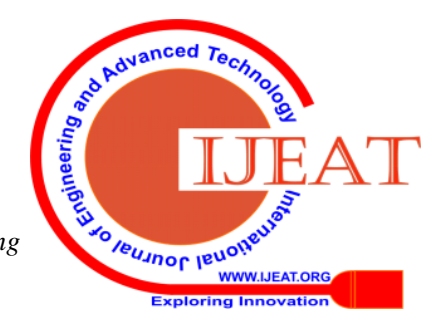

